\title{
A two-stage sodium thermal electrochemical converter: Parametric optimization and performance enhancement
}

\author{
Wanli Peng ${ }^{\mathrm{a}, \mathrm{b}}$, Julian Gonzalez-Ayala ${ }^{\mathrm{b}, \mathrm{c}}$, Shanhe Su ${ }^{\mathrm{a}}$, Jincan Chen ${ }^{\mathrm{a}, *}$, Antonio \\ Calvo Hernández ${ }^{\mathrm{b}, \mathrm{c}, * *}$ \\ a Department of Physics, Xiamen University, Xiamen, 361005, People's Republic of China \\ ${ }^{\mathrm{b}}$ Departamento de Física Aplicada, Universidad de Salamanca, 37008, Salamanca, Spain \\ ${ }^{\mathrm{c}}$ Instituto de Física Fundamental y Matemáticas, Universidad de Salamanca, 37008, Salamanca, Spain
}

\section{H I G H L I G H T S}

- A two-stage sodium thermal electrochemical converter is updated.

- Main irreversible losses are considered and the coupling of two stages is optimized.

- The maximum efficiency and power output density increase $17.5 \%$ and $40.6 \%$.

- The optimum selection criteria of main parameters are supplied.

- The optimum energetic space is given by the Pareto front.

\section{A R T I C L E I N F O}

\section{Keywords:}

Two-stage sodium thermal electrochemical converter

Main irreversible loss

Optimum operation state

Pareto front

Parametric selection criterion

\begin{abstract}
A B S T R A C T
An asymmetric two-stage sodium thermal electrochemical converter and its optimum performance are studied by means of an improved analytical model including the main losses in the overall system. Based on the study of a single-stage sodium thermal electrochemical converter, the inner process is divided into two stages including one at the $1300 \mathrm{~K}$ temperature (evaporator) and the other at the 800-1300 K intermediate temperature with the aim of improving efficiency. The parametric optimum selection criteria of a few main parameters of the two-stage device are provided and the coupling of the separate stages in an overall optimum system in terms of the appropriate intermediate temperature is particularly stressed. The maximum efficiency of the proposed overall system can attain $36.2 \%$, which is $17.5 \%$ higher than that of the best performing single-stage device, and increase up to $34.1 \%$ and $24.8 \%$ over the existing two-stage devices designed by two research groups, respectively. The Pareto front obtained from numerical multiobjective and multiparametric methods endorses previous findings and visually presents the space of the states and the energetic properties of the overall arrangement compared with the corresponding data for the isolated first and second stages.
\end{abstract}

\section{Introduction}

A sodium thermal electrochemical converter (Na-TEC), which is one promising thermally regenerative electrochemical system, converts directly heat into electricity by the isothermal expansion of sodium ions through a $\beta^{\prime \prime}$-alumina solid-electrolyte (BASE) allowing for $\mathrm{Na}^{+}$ion transport in a wide temperature range [1,2]. The emerging Na-TEC [also named as the alkali metal thermal electric converter (AMTEC)] can be traced back to the 1960s, and the technology, primarily for the NASA
Pluto/Express spacecraft mission [3], attracted extensive attention in the 1990-2000s for space applications. Some seminal studies on Na-TEC systems have been reported. For example, Weber [4] presented a theoretical analysis and described the construction of a model beta-alumina thermoelectric generator. Cole [2] studied the thermodynamic operating principles and gave some crucial expressions including current-voltage relation and efficiency. Williams et al. investigated the kinetics and transport at electrodes including the interfacial impedance model [5] and the dependence of the interfacial impedance of $\mathrm{Na}$ (g)/porous Mo/Na-Beta-double prime alumina on temperature [6]. It is

\footnotetext{
* Corresponding author.

** Corresponding author. Departamento de Física Aplicada, Universidad de Salamanca, 37008 Salamanca, Spain.

E-mail addresses: jcchen@xmu.edu.cn (J. Chen), anca@usal.es (A.C. Hernández).
} 


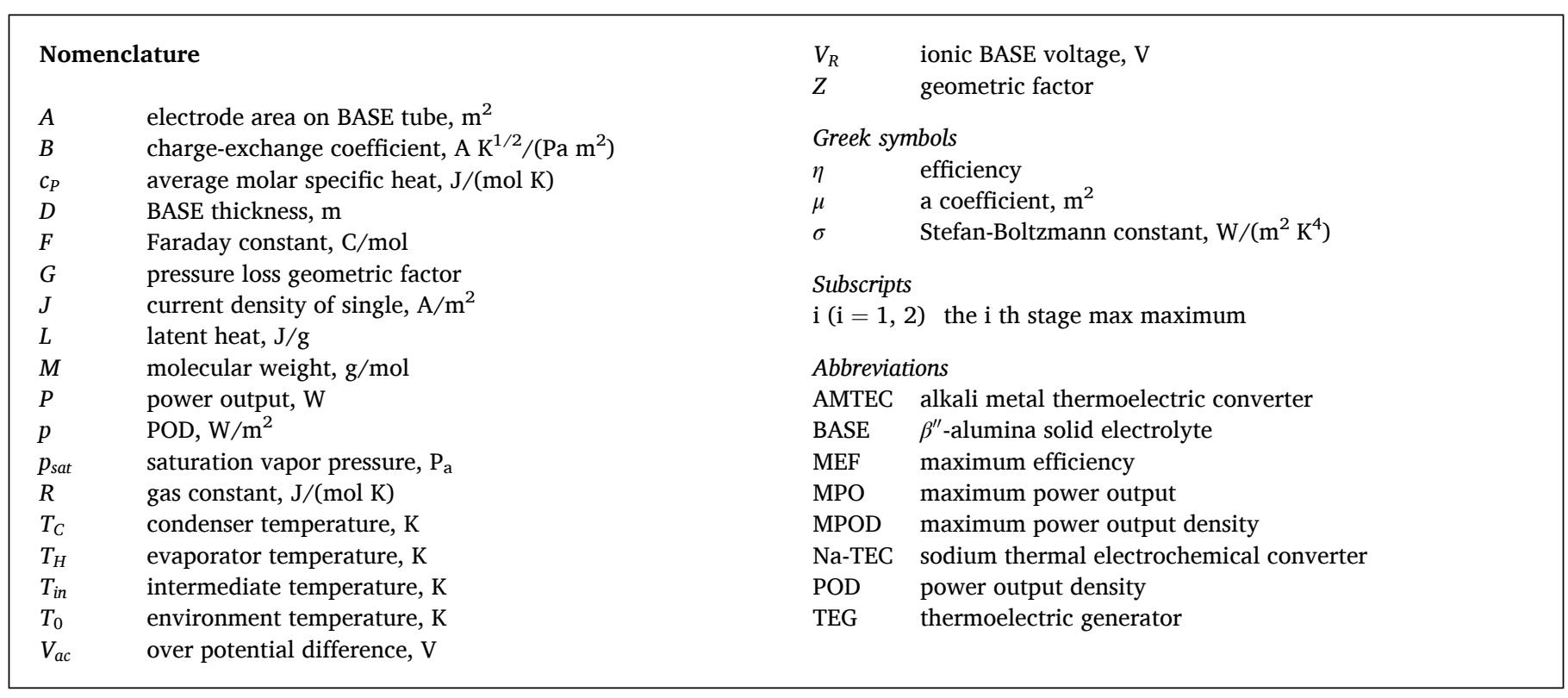

well known [7] that the efficiency of the most advanced heat engines can reach about $40 \%$ currently. Heat is directly converted to work in the isothermal ion expansion process in the Na-TEC, which can be modelled as a heat engine. In comparison, the ideal efficiency of the Na-TEC can be achieved approximately $92-95 \%$ of the efficiency of the Carnot heat engine operating the temperatures between 450 and $1300 \mathrm{~K}$ [8]. However, the practical efficiency of Na-TECs can only reach $20 \%$ for the systems at the high-temperature region of 1050-1150 K and low-temperature region of 550-625 K [9].

Some key technical challenges such as the degradation of the constituent materials [10], the high temperature sealing, and the electrolyte stability [11], has resulted in actual efficiency falling far short of the ideal efficiency. Many researchers carried out experimental and theoretical investigations to improve the efficiency and power output with significant results. For example, Nakata et al. [12] prepared TiN, TiC, $\mathrm{TiC} / \mathrm{TiN}$, and Mo electrodes for a Na-TEC by ceramic processing and studied their cathodic polarization characteristics at $600-800{ }^{\circ} \mathrm{C}$. Lodhi et al. [13] improved the efficiency of Na-TEC to $17.5 \%$ by changing some geometrical dimensions. Lodhi et al. [14] further dealt with the problem of power degradation in some detail and proposed some suggested measures to diminish loss of power with time. Lodhi et al. also investigated temperature dependent of grain growth model for Na-TEC electrodes [15] and BASE performance in an AMTEC [16]. El Genk et al. [17] identified a number of design changes to improve the performance of PX-series cells, such as increasing the BASE tube number and the BASE electrode length, developing corrugated BASE tubes, and using highly thermally conductive refractory metals (Ni or Mo) on the cell hot side. Peng et al. [18] discussed the dependence of the performance of the AMTEC on the BASE thickness and obtained the maximum efficiency (MEF) and maximum power output density (MPOD), which are, respectively, $22.3 \%$ and $6.83 \times 10^{3} \mathrm{~W} / \mathrm{m}^{2}$ at the evaporator temperature of $1170 \mathrm{~K}$. Some coupled systems have been proposed to improve the whole system performance by utilizing the AMTEC exhaust heat. For example, Wu et al. [19] displayed the mathematical model of the AMTEC-thermoelectric generator (TEG) integrated system and obtained the efficiencies of $27.4 \%$ and $31.3 \%$ for the AMTEC and AMTEC/TEG, respectively. Peng et al. [20] further extended the AMTEC/TEG integrated system and found that MEF and MPOD could climb to $34.6 \%$ and $6.43 \times 10^{4} \mathrm{~W} / \mathrm{m}^{2}$ with an increment of $9.15 \%$ and $34.0 \%$ over that of the single AMTEC. Later on, a novel coupled system comprised of a Na-TEC and a Brayton heat engine was proposed by Peng et al. [21]. The MEF and MPOD of the system attain, respectively, 41.7\% and $116 \times 10^{3} \mathrm{~W} / \mathrm{m}^{2}$, which increase $44.8 \%$ and $158 \%$ compared with that of the AMTEC working alone. In recent years, some researchers devoted themselves to improve the performance of a Na-TEC from a novel perspective by increasing the number of stages of the Na-TEC. For example, Balagopal et al. [22] provided a multi-stage sodium heat engine including at least two-stage and an electrical circuit operatively to convert thermal energy to electrical energy. Limia et al. [9] demonstrated the first time that a two-stage Na-TEC can increase the efficiency of $8 \%$ over the best performing single-stage device, and they further presented a thermal design of an asymmetric two-stage Na-TEC and achieved a $29 \%$ MEF and a $125 \mathrm{~W}$ maximum power output (MPO) [23].

Herein, we further demonstrate that the two-stage Na-TEC viewed as a series of engines [24,25] is more efficiently than already existed most efficient single Na-TEC and the former can improve the MEF and POD by up to $17.5 \%$ and $40.6 \%$ over the latter. Moreover, the MEF of the two-stage Na-TEC can improve $34.1 \%$ and $24.8 \%$ than that of two-stage Na-TECs reported in Refs. [9,23], respectively. In particular, the goal of this paper is twofold: a) to improve the model of a two-stage Na-TEC, and $b$ ) to investigate the optimum performance on the basis of the key parameters in regards with design and main losses: the BASE thickness, the current density, and the intermediate temperature between the two stages. Goal a) mainly relies on of the assumption of two single AMTEC coupled according to constraints imposed by the continuity and energy conservation (i.e., without heat leaks to the environment) so that the second AMTEC device works with heat delivered by the first one. For both of them heat transfer by conduction and radiation, and pressure drops through the porous electrodes by appropriate geometrical factors are considered. The additional assumption of the intermediate temperature for both stages rests on the work by Limia et al. [9] to minimize losses from overpotential and Ohmic contributions. As it will explained below, such an intermediate temperature could play a significant role in the performance regimes of the individual and collective behaviours. Goal b) focus in the analysis of the influence of some key parameters (as the extreme temperatures, current densities, cross sectional areas, and thickness BASEs) on the selected figures of merit: the power output density and thermal efficiency of the overall device. Thus, special attention is paid on the role of the intermediate temperature as it is found that only in a quite narrow interval of values the figures of merit achieve their corresponding maxima values.

Goals a) and b) are, respectively, accomplished in sections 2 and 3, where the performance regimes of the MEF and MPOD are analysed in terms of the optimum ranges of several key parameters. Section 4 
contains a closer analysis on the influence of the intermediate temperature. Section 5 collects the main results obtained and a comparison with previous ones. Section 6 is committed to give a complementary perspective on configurations leading to the useful information of the optimal functions and parameters using a multiobjective and multiparametric optimization which is built on the Pareto front. Finally, Section 7 is devoted to conclusions and some perspectives of future plus the pressure drop produced in the process of the recombination of electrons and ions and then flow through the porous electrode at the close circuit. The sodium vapor releases the heat $q_{\text {in }}$ and cools at constant pressure, which is regenerated in full in the next stage Na-TEC. The sodium goes through the isothermal expansion process from the intermediate plenum to the low temperature condenser plenum in which the sodium cools and condenses to discharge the waste heat, $q_{C}$.

$\eta_{1}=\frac{P_{1}}{q_{H}}=\frac{J_{1} A_{1}\left[\frac{R T_{H}}{F} \ln \left[1+p_{s a t}\left(T_{H}\right) \mu D_{1} /\left(R T_{H}\right)\right]-V_{a c, 1}-V_{R, 1}\right]}{J_{1} A_{1}\left[\frac{R T_{H}}{F} \ln \left[1+p_{s a t}\left(T_{H}\right) \mu D_{1} /\left(R T_{H}\right)\right]-V_{a c, 1}-V_{R, 1}\right]+J_{1} A_{1} M L / F+J_{1} A_{1} c_{P}\left(T_{H}-T_{i n}\right) M / F+A_{1} \sigma\left(T_{H}^{4}-T_{i n}{ }^{4}\right) / z}$,

works.

\section{The description of a two-stage Na-TEC}

The basic thermodynamic operating principles for a single Na-TEC were introduced [18]. The operating principles of a two-stage Na-TEC are similar to those of a single Na-TEC and its schematic diagram is shown in Fig. 1. $q_{H}$ is the heat flow from the heat source to the evaporator plenum of the first stage Na-TEC at temperature $T_{H}$. $q_{C}$ is the heat flow from the condenser plenum of the second stage Na-TEC at temperature $T_{C}$ to the environment at temperature $T_{0} . T_{\text {in }}$ is the temperature of the intermediate temperature plenum. $A_{1,2}$ corresponds to the first/second stage electrolyte area, $D_{1,2}$ is the first/second stage electrolyte thickness, and $P_{1,2}$ is the power output of the first/second stage Na-TEC, respectively. $P=P_{1}+P_{2}$ is the total power output of the two-stage Na-TEC device.

In the first stage operation, liquid sodium is heated and vaporized in the high-temperature plenum. Sodium cations expand isothermally though the BASE from the high-temperature plenum into the intermediate plenum where the pressure is the vapor pressure at open circuit

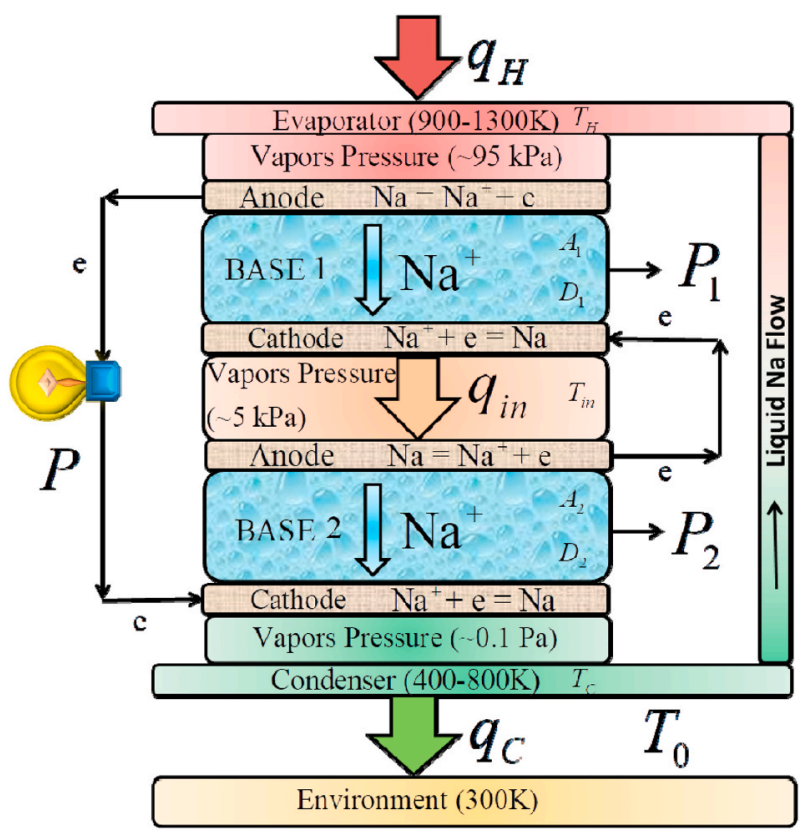

Fig. 1. The schematic diagram of a two-stage Na-TEC.

\subsection{The efficiency of the first stage Na-TEC}

The thermodynamic operation of the first stage Na-TEC is like that of a single Na-TEC. Thus, the first stage thermal first-law based efficiency, defined as the ratio of the total power output to total heat input, $\eta_{1}$ is given by [18]

where $[14,26]$

$$
V_{R, 1}=J_{1} D_{1}\left[1.62 \times 10^{-5} T_{H} \exp \left(-45.5 / T_{H}\right)+1.55 \times 10^{-7} T_{H} \exp \right.
$$
$\left.\left(3722 / T_{H}\right)\right]$,

$V_{a c, 1}=-\frac{2 R T_{H}}{F} \ln \left[\frac{1}{2}\left[\frac{J_{1}^{2} T_{H}}{B_{1}{ }^{2} p_{s a t}^{2}\left(T_{H}\right)}+4\right]^{\frac{1}{2}}-\frac{1}{2} \frac{J_{1} \sqrt{T_{H}}}{B_{1} p_{s a t}\left(T_{H}\right)}\right]+$

$\frac{2 R T_{H}}{F} \ln \left[\frac{1}{2}\left[\frac{J_{1}^{2} \sqrt{T_{H} T_{\text {in }}}}{B_{1}^{2} p_{\text {sat }}\left(T_{H}\right) p_{\text {sat }}\left(T_{\text {in }}\right)}+4\left[1+\frac{\left(1+\frac{3 G_{1}}{8 \pi}\right) \sqrt{2 \pi M R T_{H} T_{\text {in }}} \frac{J_{1}}{F}}{p_{\text {sat }}\left(T_{\text {in }}\right) \sqrt{T_{H}}}\right]\right]^{\frac{1}{2}}\right.$

$\left.+\frac{1}{2} \frac{J_{1}\left(T_{H} T_{\text {in }}\right)^{\frac{1}{4}}}{B_{1} \sqrt{p_{\text {sat }}\left(T_{H}\right) p_{\text {sat }}\left(T_{\text {in }}\right)}}\right]$,

$P_{\text {sat }}(T)=10^{9.678-5383.2 / T}$ is the saturation vapor pressure at temperature $T$ [14], $V_{R, 1}$ is the first stage ionic BASE voltage, $V_{a c, 1}$ is the first stage over potential difference, $G_{1}$ is the first stage pressure loss geometric factor, $B_{1}$ denotes the first stage charge-exchange coefficient, $J_{1}$ the first stage current density, $L$ and $M$ are the sodium vaporization latent heat and molecular weight, $c_{P}$ is the molar specific heat, $z$ is the radiation reduction factor, $F$ the Faraday constant, $\sigma$ the Stefan-Boltzmann constant, $\mu$ is a coefficient, and $R$ is the gas constant. The specific relational expression among $T_{H}, \mu, D_{1}$, and $T_{\text {in }}$ should satisfy the following relation [26]

$\frac{p_{\text {sat }}\left(T_{H}\right)}{1+p_{\text {sat }}\left(T_{H}\right) \mu D_{1} /\left(R T_{H}\right)}=p_{\text {sat }}\left(T_{\text {in }}\right) \sqrt{\frac{T_{H}}{T_{\text {in }}}}$.

\subsection{The efficiency of the second stage Na-TEC}

As shown in Fig. 1, the heat $q_{\text {in }}$ from the first stage is fully utilized by the second stage Na-TEC. The thermal efficiency $\eta_{2}$ can be written as

$\eta_{2}=\frac{P_{2}}{q_{\text {in }}}=\frac{J_{2} A_{2}\left[\frac{R T_{\text {in }}}{F} \ln \left[1+p_{\text {sat }}\left(T_{\text {in }}\right) \mu D_{2} /\left(R T_{\text {in }}\right)\right]-V_{a c, 2}-V_{R, 2}\right]}{J_{1} A_{1} M L / F+J_{1} A_{1} c_{P}\left(T_{H}-T_{\text {in }}\right) M / F+A_{1} \sigma\left(T_{H}^{4}-T_{\text {in }}{ }^{4}\right) / z}$,

where in this case [14,26]

$V_{R, 2}=J_{2} D_{2}\left[1.62 \times \quad 10^{-5} T_{\text {in }} \exp \left(-45.5 / T_{\text {in }}\right)+1.55 \times\right.$ $\left.10^{-7} T_{\text {in }} \exp \left(3722 / T_{\text {in }}\right)\right]$, 


$$
\begin{aligned}
& V_{a c, 2}=-\frac{2 R T_{\text {in }}}{F} \ln \left[\frac{1}{2}\left[\frac{J_{2}^{2} T_{\text {in }}}{B_{2}{ }^{2} p_{\text {sat }}^{2}\left(T_{\text {in }}\right)}+4\right]^{\frac{1}{2}}-\frac{1}{2} \frac{J_{2} \sqrt{T_{\text {in }}}}{B_{2} p_{\text {sat }}\left(T_{\text {in }}\right)}\right]+ \\
& \frac{2 R T_{\text {in }}}{F} \ln \left[\frac{1}{2}\left[\frac{J_{2}{ }^{2} \sqrt{T_{\text {in }} T_{C}}}{B_{2}^{2} p_{\text {sat }}\left(T_{\text {in }}\right) p_{\text {sat }}\left(T_{C}\right)}+4\left[1+\frac{\left(1+\frac{3 G_{2}}{8 \pi}\right) \sqrt{2 \pi M R T_{\text {in }} T_{C}} \frac{J_{2}}{F}}{p_{\text {sat }}\left(T_{C}\right) \sqrt{T_{\text {in }}}}\right]\right]^{\frac{1}{2}}\right.
\end{aligned}
$$

$\left.+\frac{1}{2} \frac{J_{2}\left(T_{i n} T_{C}\right)^{\frac{1}{4}}}{B_{2} \sqrt{p_{\text {sat }}\left(T_{\text {in }}\right) p_{\text {sat }}\left(T_{C}\right)}}\right], V_{R, 2}$ indicates the second stage ionic BASE

voltage, $V_{a c, 2}$ is the second stage over potential difference, $G_{2}$ means the second stage pressure loss geometric factor, $J_{2}$ indicates the second stage current density, and $B_{2}$ stands for the second stage charge-exchange coefficient. Similarly, some parameters including $T_{i n}, \mu, D_{2}$, and $T_{C}$ should satisfy the following equation [26]

$\frac{p_{\text {sat }}\left(T_{\text {in }}\right)}{1+p_{\text {sat }}\left(T_{\text {in }}\right) \mu D_{2} /\left(R T_{\text {in }}\right)}=p_{\text {sat }}\left(T_{C}\right) \sqrt{\frac{T_{\text {in }}}{T_{C}}}$.

\section{The MEF and MPOD of the two-stage system}

Eqs. (5) and (6) indicate that both $\eta$ and $P$ of the two-stage system depend on several crucial parameters including $T_{H}, T_{i n}, T_{C}, J_{1}, J_{2}, A_{1}, A_{2}$, $D_{1}$, and $D_{2}$ (some other parameters not involved in the optimization have been fixed to typical values for this analysis, see Table 1). The last four of them involve an optimization on the design since they are related to the BASE geometry. It is convenient to introduce the power output density (POD), i.e., $p=P / A_{1}$, so that the area ratio $A_{1} / A_{2}$ may be taken as a parameter. $T_{H}$ can be fixed to the maximum attainable value, which is also listed in Table 1 . The remainder can be considered as operation variables. Among these variables, besides the constraint conditions given by Eqs. (2) and (4), there also exist two constraint relations imposed by the current continuity and energy conservation as follows

$J_{1} A_{1}=J_{2} A_{2}$

and

$J_{1} A_{1} c_{P}\left(T_{H}-T_{\text {in }}\right) M / F+J_{1} A_{1} M L / F+A_{1} \sigma\left(T_{H}^{4}-T_{i n}{ }^{4}\right) / z=P_{2}+J_{2} A_{2} c_{P}\left(T_{\text {in }}-T_{C}\right) M / F+$

$J_{2} A_{2} M L / F+A_{2} \sigma\left(T_{i n}^{4}-T_{C}^{4}\right) / z$

\subsection{The efficiency of the two-stage Na-TEC}

From Eqs. (1) and (3), the thermal efficiency, $\eta$, of the two-stage system can be expressed as

$\eta=\frac{P}{q_{H}}=\eta_{1}+\eta_{2}\left(1-\eta_{1}\right)$

where the power output of the whole device is,

$$
\begin{aligned}
P & =P_{1}+P_{2}=q_{H}-J_{2} A_{2} M L / F-J_{2} A_{2} c_{P}\left(T_{\text {in }}-T_{C}\right) M / F-A_{2} \sigma\left(T_{\text {in }}^{4}-T_{C}^{4}\right) / z \\
& =J_{1} A_{1}\left[\frac{R T_{H}}{F} \ln \left[1+p_{\text {sat }}\left(T_{H}\right) \mu D_{1} /\left(R T_{H}\right)\right]-V_{a c, 1}-V_{R, 1}\right]+ \\
& J_{2} A_{2}\left[\frac{R T_{\text {in }}}{F} \ln \left[1+p_{\text {sat }}\left(T_{\text {in }}\right) \mu D_{2} /\left(R T_{\text {in }}\right)\right]-V_{a c, 2}-V_{R, 2}\right]
\end{aligned}
$$

The particular cases addressed in sections 2.1 and 2.2 can be recovered from the above equations with the appropriate selection of parameters (see Appendix A).

Table 1

Parameters used in the two-stage Na-TEC [2,9,27].

\begin{tabular}{ll}
\hline$T_{H}(\mathrm{~K})$ & 1300 \\
$B_{1}\left(\mathrm{~A} \mathrm{~K} \mathrm{~K}^{1 / 2} /\left(\mathrm{P}_{\mathrm{a}} \mathrm{m}^{2}\right)\right)$ & 50 \\
$B_{2}\left(\mathrm{~A} \mathrm{~K}^{1 / 2} /\left(\mathrm{P}_{\mathrm{a}} \mathrm{m}^{2}\right)\right)$ & 600 \\
$F(\mathrm{C} / \mathrm{mol})$ & 96485 \\
$c_{P}(\mathrm{~J} /(\mathrm{g} \mathrm{K}))$ & 1.285 \\
$\mu\left(\mathrm{m}^{2}\right)$ & $10^{7}$ \\
$L(\mathrm{~J} / \mathrm{g})$ & 4480 \\
$M(\mathrm{~g} / \mathrm{mol})$ & 23 \\
$G_{1}$ & 10 \\
$G_{2}$ & 10 \\
$\sigma\left(\mathrm{W} /\left(\mathrm{m}^{2} \mathrm{~K}^{4}\right)\right)$ & $5.67 \times 10^{-8}$ \\
$Z$ & 50 \\
$R(\mathrm{~J} /(\mathrm{mol} \mathrm{K}))$ & 8.314 \\
\hline
\end{tabular}

According to the analyses above, there are only three independent variables for the two-stage Na-TEC shown in Fig. 1. For example, we can choose $T_{\text {in }}, D_{2}$, and $J_{1}$ as independent variables to optimize the performance of the two-stage Na-TEC. When $T_{i n}$ is a given value, Eqs. (5) and (6) and the parameter values given in Table 1 can be used to generate the 3D-graphs of the efficiency $\eta$ and POD $p$ varying with $J_{1}$ and $D_{2}$, as displayed in Fig. 2(a) and (b), respectively. It is observed from Fig. 2 that there exist the different optimal values of $J_{1}$ and $D_{2}$ to maximize $\eta$ and $p$. When $D_{2}$ is optimally chosen, the influences of $J_{1}$ on both $\eta$ and $p$ are shown in Fig. 3(a), where $\eta_{\max , T_{i n}}$ and $p_{\max , T_{\text {in }}}$ are the local MEF and MPOD of the two-stage Na-TEC for a given $T_{i n}, p_{\eta_{\max }, T_{\text {in }}}$ and $\eta_{p_{\max }, T_{\text {in }}}$ are the POD at the local MEF and the efficiency at the local MPOD, respectively, and $J_{1, \eta_{\max }, T_{\text {in }}}$ and $J_{1, p_{\max }, T_{\text {in }}}$ are the optimal values of $J_{1}$ at the local MEF and MPOD. Fig. 3(a) displays that there are a local MEF $\eta_{\max , T_{\text {in }}} \approx 36.2 \%$ increasing about $34.1 \%$ and $24.8 \%$ compared with that of the two-stage Na-TECs mentioned in Refs. [9,23], respectively, and a local MPOD $p_{\max , T_{\text {in }}} \approx 40.6 \times 10^{3} \mathrm{~W} / \mathrm{m}^{2}$. The $\tilde{\eta p}$ characteristic curve depicted in Fig. 3(b) shows more clearly the optimally operating region of the two-stage Na-TEC. In the ranges of $p<p_{\eta_{\max }, T_{\text {in }}}$ and $\eta<\eta_{p_{\max }, T_{\text {in }},}, p$ increases with the increase of $\eta$. Thus, the system should be operated in the ranges of

$\eta_{p_{\max }, T_{\text {in }}} \leq \eta \leq \eta_{\max , T_{\text {in }}}$

and

$p_{\eta_{\max }, T_{i n}} \leq p \leq p_{\max , T_{i n}}$.

The optimal range of $J_{1}$ can be also determined by

$J_{1, \eta_{\max }, T_{\text {in }}} \leq J_{1} \leq J_{1, p_{\max }, T_{\text {in }}}$,

as it can be seen from Fig. 3(a).

\section{Effects of $T_{\text {in }}$}

In the above section, the intermediate temperature $T_{\text {in }}$ was taken as a constant. However, this temperature plays a fundamental role in the overall performance of the two-stage Na-TEC. Below, we will analyse in detail the influences of $T_{\text {in }}$ on the optimized regimes of the efficiency and 

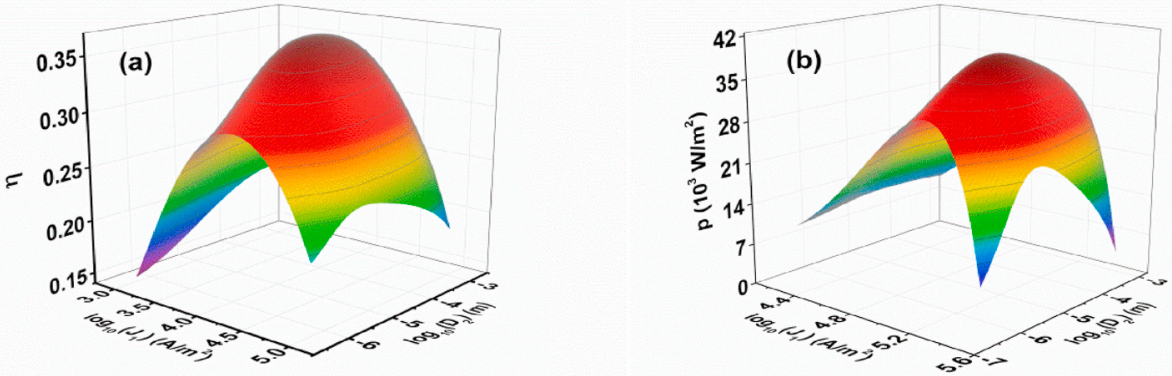

Fig. 2. (a) $\eta$ and (b) $p$ as functions of $J_{1}$ and $D_{2}$ at $T_{i n}=800 \mathrm{~K}$.
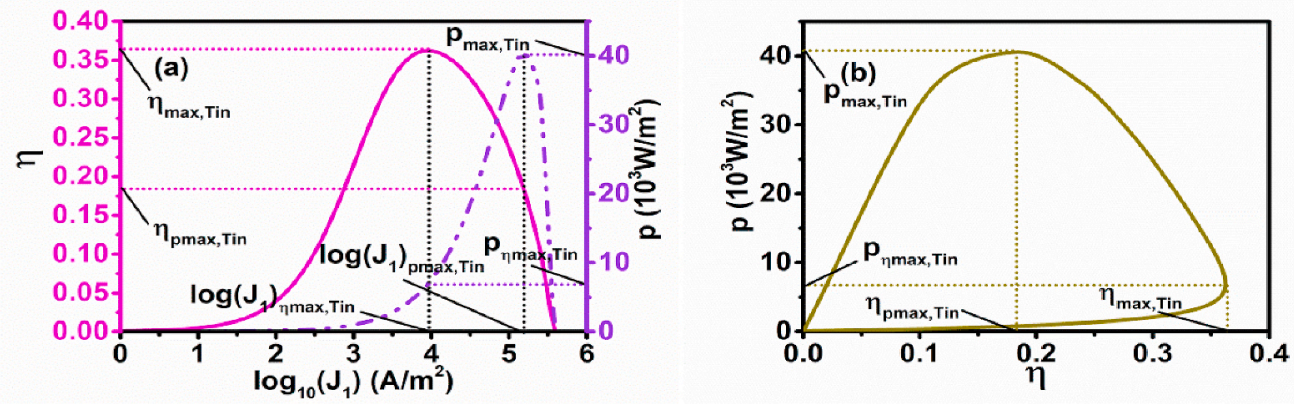

Fig. 3. (a) $\eta$ and $p$ as functions of $J_{1}$ and (b) the curve of $p$ versus $\eta$ at $T_{\text {in }}=800 \mathrm{~K}$, where $D_{2}$ has been optimized.

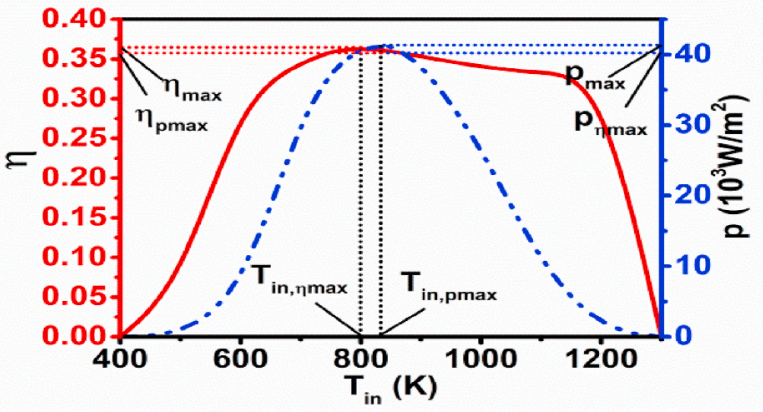

Fig. 4. $\eta$ and $p$ as functions of $T_{i n}$, where $J_{1}$ and $D_{2}$ are such that it optimizes efficiency and POD.

POD.

Eqs. (5) and (6) can be further used to discuss the effects of $T_{\text {in }}$ on both $\eta_{\max , T_{\text {in }}}$ and $p_{\max , T_{\text {in }}}$, as shown in Fig. 4, where $J_{1}$ and $D_{2}$ have been optimized, $\eta_{p_{\max }}$ and $p_{\eta_{\max }}$ are the optimal values of $\eta$ at the MPOD and $p$ at the MEF, and $T_{i n, \eta}$ and $T_{i n, p}$ are the optimal values of $T_{\text {in }}$ at the MEF and MPOD. When $T_{\text {in }}=800 \mathrm{~K} \equiv T_{i n, \eta}$, the system attains its MEF, i.e., $\eta_{\max }=$ $36.2 \%$. When $T_{i n}=840 \mathrm{~K} \equiv T_{i n, p}$, the system attains its MPOD, i.e., $p_{\max }=41.2 \times 10^{3} \mathrm{~W} / \mathrm{m}^{2}$. The optimal region of $T_{\text {in }}$ can be determined by

$T_{\text {in, } \eta_{\max }} \leq T_{\text {in }} \leq T_{\text {in. } p_{\max }}$,

and the optimal regions of $\eta$ and $p$ are then re-expressed as

$\eta_{p_{\max }} \leq \eta \leq \eta_{\max }$

$p_{\eta_{\max }} \leq p \leq p_{\max }$.

It is noteworthy that both the efficiency and the POD are capable of achieving considerable values when $T_{\text {in }}$ satisfies Eq. (12). The optimal range of $T_{\text {in }}$ isn't large and special attention should be paid to this problem in the design of two-stage Na-TECs.

When the two-stage Na-TEC is operated at the optimal states, which are in the ranges satisfied by Eqs. (13) and (14), the optimum values of $T_{C}, A_{1} / A_{2}, J_{1}, J_{2}, D_{1}$, and $D_{2}$, which are represented by $T_{C, \eta_{\max }}$ and $T_{C, p_{\max }}$, $\left(A_{1} / A_{2}\right)_{\eta_{\max }}$ and $\left(A_{1} / A_{2}\right)_{p_{\max }}, J_{1, \eta_{\max }}$ and $J_{1, p_{\max }}, J_{2, \eta_{\max }}$ and $J_{2, p_{\max }}, D_{1, \eta_{\max }}$ and $D_{1, p_{\max }}, D_{2, \eta_{\max }}$ and $D_{2, p_{\max }}$, are closely dependent on $T_{i n}$, as shown in Fig. 5. $T_{C, \eta_{\max }},\left(A_{1} / A_{2}\right)_{\eta_{\max }}, J_{1, \eta_{\max }}, J_{2, \eta_{\max }}$, and $D_{2, \eta_{\max }}$ are obviously different from $T_{C, p_{\max }},\left(A_{1} / A_{2}\right)_{p_{\max }}, J_{1, p_{\max }}, J_{2, p_{\max }}$, and $D_{2, p_{\max }}$. The shaded regions between the red lines and the blue dash dot dots are the optimized regions of $T_{C}$, $A_{1} / A_{2}, J_{1}, J_{2}$, and $D_{2}$, which are determined by.

$T_{C, \eta_{\max }} \leq T_{C} \leq T_{C, p_{\max }}$,

$\left(A_{1} / A_{2}\right)_{p_{\max }} \leq A_{1} / A_{2} \leq\left(A_{1} / A_{2}\right)_{\eta_{\max }}$,

$J_{1, \eta_{\max }} \leq J_{1} \leq J_{1, p_{\max }}$,

$J_{2, \eta_{\max }} \leq J_{1} \leq J_{2, p_{\max }}$,

and

$D_{2, p_{\max }} \leq D_{2} \leq D_{2, \eta_{\max }}$,

as shown in Fig. 5(a-d) and (f), respectively. It is clearly seen from Eq. (2) that $D_{1}$ depends only on $T_{\text {in }}$ and $T_{H}$. For the given values of $T_{i n}$ and $T_{H}, D_{1}$ is a determinate quantity, which is independent of the state of the MEF or MPOD, so that $D_{1, \eta_{\max }}$ and $D_{1, p_{\max }}$ are the same, as indicated in Fig. 5(e).

\section{Results and comparison with previous works}

When $T_{\text {in }}=T_{H}$ or $T_{\text {in }}=T_{C}$, one can obtain the expressions of the efficiency $\eta$ and power output $P$ of a single Na-TEC (see Appendix A), i.e. [18]

and 

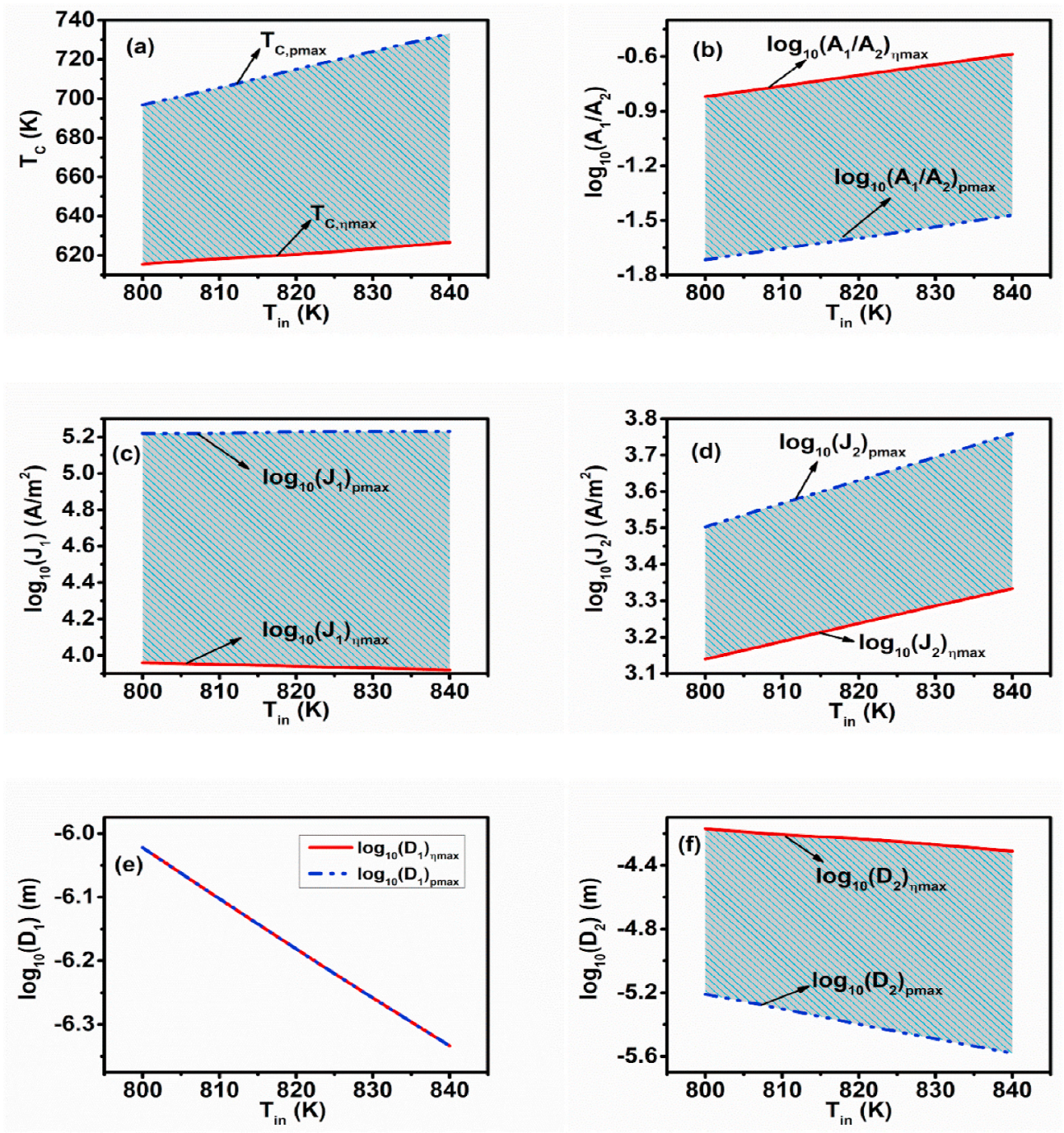

Fig. 5. (a) $T_{C}$, (b) $A_{1} / A_{2}$, (c) $J_{1}$, (d) $J_{2}$, (e) $D_{1}$ and (f) $D_{2}$ as functions of $T_{i n}$.

$P=J A\left[\frac{R T_{H}}{F} \ln \left[1+p_{s a t}\left(T_{H}\right) \mu D /\left(R T_{H}\right)\right]-V_{a c}-V_{R}\right]$,

different results are in order at this point.

Firstly, the comparison between operation of the single- and dual-

$\eta=\frac{J\left[\frac{R T_{H}}{F} \ln \left[1+p_{s a t}\left(T_{H}\right) \mu D /\left(R T_{H}\right)\right]-V_{a c}-V_{R}\right]}{J\left[\frac{R T_{H}}{F} \ln \left[1+p_{s a t}\left(T_{H}\right) \mu D /\left(R T_{H}\right)\right]-V_{a c}-V_{R}\right]+J M L / F+J c_{P}\left(T_{H}-T_{C}\right) M / F+\sigma\left(T_{H}^{4}-T_{C}^{4}\right) / z}$,

By using Eqs. (5), (6), (20) and (21), we can plot the optimized curves of the efficiency versus the POD of a two-stage Na-TEC and a single NaTEC operating at the same temperature span, as indicated by Fig. 6. As can be observed, the optimized performance of a two-stage Na-TEC is better than that of a single Na-TEC. The MEF and MPOD of a two-stage Na-TEC are $36.2 \%$ and $41.2 \times 10^{3} \mathrm{~W} / \mathrm{m}^{2}$, while the MEF and MPOD of a single Na-TEC are only $30.8 \%$ and $29.3 \times 10^{3} \mathrm{~W} / \mathrm{m}^{2}$ [18]. The MEF and MPOD of the former increase $17.5 \%$ and $40.6 \%$ compared with those of the latter. Besides, the MEF of the two-stage Na-TEC mentioned here increase $34.1 \%$ and $24.8 \%$ compared with the MEFs $27.0 \%$ and $29.0 \%$ of two-stage Na-TECs reported in Refs. $[9,23]$. This shows that the performance of two-stage Na-TECs can be largely improved through the optimum design. Two additional comments on the comparison of stage Na-TEC can be done mainly under two different conditions: at the same temperature of the external reservoirs, on one side, and a fixed heat input and a fixed hot-side temperature, on the other side. In the case of the same extreme temperatures a comparison is presented in Fig. 6 (inner curve) where it is appreciated clearly as the loop-like of density power versus efficiency is the same but in the single stage case both efficiency as power density have significant smaller values $\left(29.3 \times 10^{3}\right.$ $\mathrm{W} / \mathrm{m}^{2}$ and $30.8 \%$ ) compared with those obtained in the two-stage case $\left(41.2 \times 10^{3} \mathrm{~W} / \mathrm{m}^{2}\right.$ and $\left.36.2 \%\right)$. Under different temperatures ranges results may significantly differ. For instance, in Ref. [18] values for a single AMTEC with high temperature of $1170 \mathrm{~K}$ and the optimized values were found to be around $7000 \mathrm{~W} / \mathrm{m}^{2}$ and $23 \%$ for maximum power density and efficiency, respectively, although small variations 


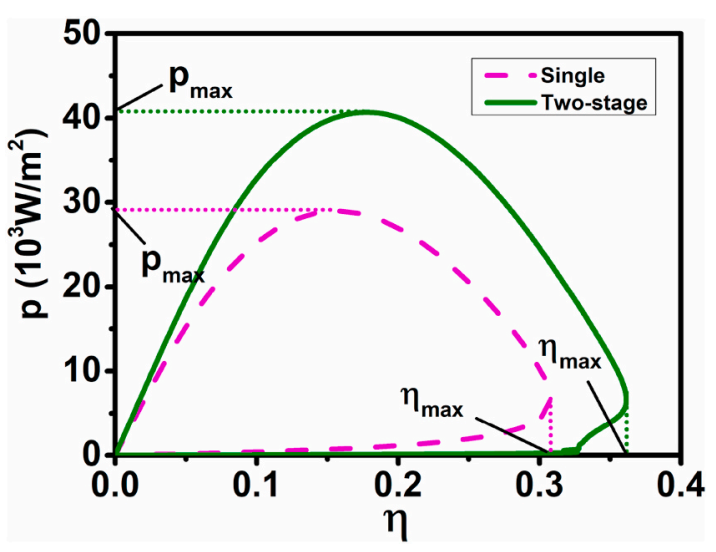

Fig. 6. The optimized curves of efficiency versus the POD for a two-stage NaTEC and a single Na-TEC operating at the same temperature span.

can be produced depending on the remainder parameter being optimized (current density or thickness of BASE).

Secondly, it should be noted that a proper comparison of the values for power output and efficiency here presented with those of previous two-stage works $[9,23]$ is not straightforward, both for the different theoretical point of view and for the mathematical methods. In Ref. [23] the reported maximum efficiency was $29 \%$ (with $4500 \mathrm{~A} / \mathrm{m}^{2}<J_{1}<$ $5500 \mathrm{~A} / \mathrm{m}^{2}$ ) and a maximum power output of $125 \mathrm{~W}$. In that case conduction bypass through the Na-TEC liquid-return path, corrugated geometries with radiation shields were considered by a mathematical finite-element model. In Refs. [9] the MEF reported is $27 \%$ at $T_{\text {in }}$ around $775 \mathrm{~K}$ with $J_{1}=3500 \mathrm{~A} / \mathrm{m}^{2}$ and $A_{1} / A_{2}=0.5$ while maximum power density is monotonically increasing with $T_{\text {in }}$, obtaining a value around $2000 \mathrm{~W} / \mathrm{m}^{2}$ at $T_{\text {in }}=1050 \mathrm{~K}$. For that case, values $T_{\text {evap }}=1150 \mathrm{~K}$ and $T_{\text {cond }}=550 \mathrm{~K}$ were considered and the model was based on numerical simulations for the conduction heat transfer through the walls and the capillary wick of the device, plus radiative heat transfer from the cathode to the condenser, where the parasitic losses are proportional to the temperature dependence between the evaporator and the condenser. Here, POD and MEF states are achieved at intermediate temperatures $T_{\text {in }}$ of $840 \mathrm{~K}$ and $800 \mathrm{~K}$, respectively (see Fig. 4) allowing to establish upper and lower bounds for optimum values. In particular, the MEF is found at $800 \mathrm{~K}$ with $J_{1}$ around $10000 \mathrm{~A} / \mathrm{m}^{2}$ (see Fig. $5 \mathrm{c}$ ) and $\mathrm{A}_{1} / \mathrm{A}_{2}$ value around 0.16 (see Fig. 5b), but now $T_{H}=1300 \mathrm{~K}$ and $T_{C}$ is also an optimized parameter with values around $620 \mathrm{~K}$ (see Fig. 5a). The maximum POD is found around $40 \mathrm{~kW} / \mathrm{m}^{2}$ at $T_{\text {in }}=840 \mathrm{~K}$ and displaying values somewhat higher for $J_{1}$ and $T_{C}$ but smaller for $A_{1} / A_{2}$. Notice that the definition of POD differs from the one considered by Limia et al. [9], defined as $P /$ $\left(A_{1}+A_{2}\right)$, meanwhile in the present case it is defined as $P / A_{1}$. Above considerations point out that the main difference between both approaches could be a consequence from the optimization scheme rather than from a structural design. For instance, in Ref. [9] a monotonous increasing power density (with respect to the intermediate temperature) is reported while the efficiency exhibits a parabolic-like behaviour with a well-defined maximum around $750 \mathrm{~K}$. This feature differs from the present case (see Fig. 4) where a parabolic-like behaviour is displayed for both power density and efficiency. Thus, the parametrization of power versus efficiency (through elimination of $T_{i n}$ ) produce the loop-like behaviour in Fig. 6, which sometimes is considered as a signature for the performance of real heat devices.

\section{Multi-parametric and multi-objective optimization predictions}

The parametric optimization of the single objective function like the efficiency or the POD of the two-stage Na-TEC has been obtained by using three independent variables $J_{1}, T_{\text {in }}$, and $D_{2}$ (directly linked to $T_{C}$ ).
Herein, Pareto front [28-30] displays the best compromise among desirable quantities where a further improvement in one function involves the degrading of the rest, which is based on numerical multi-parametric and multi-objective optimization methods. The results obtained are shown in Fig. 6. A global optimization involving the simultaneous optimization of three independent parameters $\left(J_{1}, T_{i n}\right.$, and $T_{C}$ ) and two objective functions POD and efficiency could be realized. Reasonable values for $J_{1}, T_{i n}$, and $T_{C}$ are such that $800 \mathrm{~K} \leq T_{\text {in }} \leq 1300 \mathrm{~K}$, $400 \mathrm{~K} \leq T_{C} \leq 800 \mathrm{~K}$, and $\eta, \eta_{1}, \eta_{2}, P, P_{1}$, and $P_{2}$ are all positive quantities. Simultaneously, the multi-objective optimization is completed for the three configurations including the overall system, the first-stage Na-TEC, and the second-stage Na-TEC with the purpose of comparing the optimization of them. The physical variable space is displayed in Fig. 7(a). A random search in the physical region produces a first generation of points in the $\tilde{\eta} p$ space which are mapped into the energetic space depicted in Fig. 7(b) for the isolated second-stage Na-TEC, isolated first-stage Na-TEC, and the two-stage Na- TEC system.

It is worth noting that it is impossible to obtain an optimum configuration for POD and efficiency in the isolated second-stage system because of the limited range of $J_{1}$. This is a straightforward consequence of the current continuity $J_{1} A_{1}=J_{2} A_{2}$ joined to the cut-off current $J_{1}$ (see Fig. 3(a)). Both features impose constraints on $J_{2}$ which prevent the optimum configuration of the isolated second-stage system.

In the case of isolated configuration of the first-stage Na-TEC, there is a clear compromise region between the MPOD and the MEF operation regimes. Besides, the space of parameters in Fig. 7(a) indicates a preference for temperature $T_{\text {in }} \approx 800 \mathrm{~K}$ which allows to obtain the MEF and MPOD for the first-stage Na-TEC; additionally, in a slender range for the $T_{\text {in }}$ values it can be observed a noticeable improvement in both the efficiency and POD of the whole system, in agreement with the result exhibited in Fig. 4. It is indicated that the first-stage design should be modified with a small variation not to operate in its optimum configuration, leading to an obvious improvement of the overall system performance; raising the POD up to almost $41.4 \%$ and the MEF up to almost $29.1 \%$, as shown in Fig. 7(b).

\section{Conclusions and perspectives}

A novel axisymmetric two-stage Na-TEC system including the main losses has been displayed. The matching problem between the first and second stages has been addressed with some outstanding results that can be beneficial for the development of high-performance duel-stage NaTEC systems. Several significant results are listed:

(i)The specific impact of key parameters including the BASE area ratio of the first stage to the second stage, the intermediate plenum temperature, the thickness of the electrolyte of the second stage, and the current density of the first stage have been discussed. Accordingly, the optimum selective criteria of these parameters leading to optimized upper and lower bounds of the POD and efficiency have been identified and analysed. Special emphasis has been paid on the calculated the optimized parameters and the optimal intermediate temperatures. These last values define a quite narrow interval in between which the power output density and thermal efficiency of the overall device get their respective maximum values. This point could be of some importance from a design point of view.

(ii) The energetic performance of the duel-stage Na-TEC system is better than that of the single Na-TEC, as it can be checked in the loop-like behaviour of POD versus efficiency for both configurations. Herein, the MEF and MPOD of the duel-stage Na-TEC increase about $17.5 \%$ and $40.6 \%$ compared with those of the single Na-TEC. The energetic advantages of the present system are more obvious than those of other duel-stage systems. Specifically, the MEF of the two-stage Na-TEC increase $34.1 \%$ and $24.8 \%$ compared with those of two-stage Na-TECs reported in Refs. [9, 

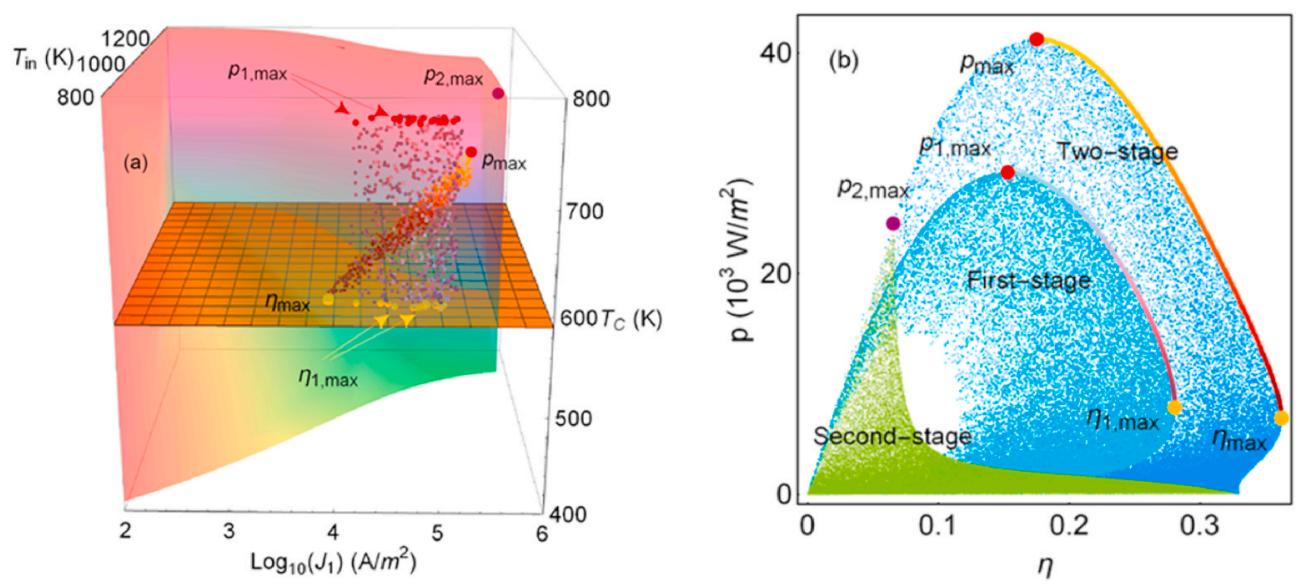

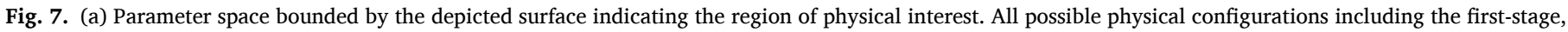
the second-stage, and the whole system are depicted. (b) Pareto front is shown by energetic representation in the $\eta^{\sim} p$ space.

23]. Thus, a two-stage Na-TEC can be chosen for applications requiring high-performance.

(iii) The multi-parametric and multi-objective optimization analysis on the basis of the Pareto front and physically available space of states indicate that the configuration of optimum performance for the first stage system matches partially that for the two-stage system; this feature is endorsed by previous results. The differences between the optimum behaviors for collective and individual systems could be explained on the basis of linear irreversible thermodynamics $[24,25]$ framework. See detail below.

The study displayed here is subject to a further extension to take into account various heat and fuel sources such as the external combustion, the nuclear reactor, the waste heat, or concentrated solar, in order to guide the design of more efficient and sustainable Na-TECs. Finally, it should be mentioned that the perspective of designing multi-step TEC beyond the two-stage model were presented [9,22]. At least theoretically, there exists linear irreversible thermodynamics models for arrays of coupled heat engines working between arbitrary temperature differences. A characteristic feature of these models is a control parameter which may select different operation regimes for the whole array without imposing the same operation regime to every engine in the array $[24,25]$. In other words, the selected intermediate temperatures could affect the more efficient operation regime in each case for both the collective and individual behaviours. Another notable feature is that these models are amenable to describe the corresponding refrigeration systems [25]. All together open novel perspectives to analyse multi-step
TEC energy systems where the bridge between the individual and collective behaviours depends on specific set of transport coefficients and the temperature profile, thus allowing a unified thermodynamic description of TECs.

\section{CRediT authorship contribution statement}

Wanli Peng: Conceptualization, Methodology, Software, Formal analysis, Writing - original draft, Writing - review \& editing. Julian Gonzalez-Ayala: Software, Formal analysis, Writing - review \& editing. Shanhe Su: Validation, Formal analysis, Visualization. Jincan Chen: Resources, Conceptualization, Writing - review \& editing, Supervision, Data curation. Antonio Calvo Hernández: Resources, Conceptualization, Writing - review \& editing, Supervision, Data curation.

\section{Declaration of competing interest}

The authors declare that they have no known competing financial interests or personal relationships that could have appeared to influence the work reported in this paper.

\section{Acknowledgments}

This work has been supported by China Scholarship Council under the State Scholarship Fund (No. 201906310095). J. G. A. acknowledges Instituto Universitario de Física Fundamental y Matemáticas. We thank the Reviewers for their valuable suggestions.

\section{Appendix A}

When $T_{\text {in }}=T_{H}$, one can delete constraint equations (7) and (8) and get $J_{1}=0, D_{1}=0, \eta_{1}=0$, and $p_{1}=0$, i.e., the first-stage is disappeared. Eqs. (5) and (6) can be written as

$\eta=\eta_{2}=\frac{J_{2}\left[\frac{R T_{H}}{F} \ln \left[1+p_{s a t}\left(T_{H}\right) \mu D_{2} /\left(R T_{H}\right)\right]-V_{a c, 2}-V_{R, 2}\right]}{J_{2}\left[\frac{R T_{H}}{F} \ln \left[1+p_{s a t}\left(T_{H}\right) \mu D_{2} /\left(R T_{H}\right)\right]-V_{a c, 2}-V_{R, 2}\right]+J_{2} M L / F+J_{2} c_{P}\left(T_{H}-T_{C}\right) M / F+\sigma\left(T_{H}^{4}-T_{C}^{4}\right) / z}$

and

$P=P_{2}=J_{2} A_{2}\left[\frac{R T_{H}}{F} \ln \left[1+p_{s a t}\left(T_{H}\right) \mu D_{2} /\left(R T_{H}\right)\right]-V_{a c, 2}-V_{R, 2}\right]$.

When $T_{\text {in }}=T_{C}$, one can delete constraint equations (7) and (8) and get $J_{2}=0, D_{2}=0, \eta_{2}=0$, and $p_{2}=0$, i.e., there does not exist the secondstage. Eqs. (5) and (6) can be written as 
$\eta=\eta_{1}=\frac{J_{1}\left[\frac{R T_{H}}{F} \ln \left[1+p_{s a t}\left(T_{H}\right) \mu D_{1} /\left(R T_{H}\right)\right]-V_{a c, 1}-V_{R, 1}\right]}{J_{1}\left[\frac{R T_{H}}{F} \ln \left[1+p_{s a t}\left(T_{H}\right) \mu D_{1} /\left(R T_{H}\right)\right]-V_{a c, 1}-V_{R, 1}\right]+J_{1} M L / F+J_{1} c_{P}\left(T_{H}-T_{C}\right) M / F+\sigma\left(T_{H}^{4}-T_{C}{ }^{4}\right) / z}$

and

$P=P_{1}=J_{1} A_{1}\left[\frac{R T_{H}}{F} \ln \left[1+p_{s a t}\left(T_{H}\right) \mu D_{1} /\left(R T_{H}\right)\right]-V_{a c, 1}-V_{R, 1}\right]$.

Obviously, Eqs. (A1)and (A2) exactly equal Eqs. (A3) and (A4), which represent the efficiency and power output of a single Na-TEC. For the sake of simplicity, subscript 2 in Eqs. (A1) and (A2) or subscript 1 in Eqs. (A3) and (A4) may be deleted so that Eqs. (20) and (21) are directly obtained from the above equations.

\section{References}

[1] Q. Wang, W. Yao, H. Zhang, X. Lu, Analysis of the performance of an alkali metal thermoelectric converter (AMTEC) based on a lumped thermal-electrochemical model, Appl. Energy 216 (2018) 195-211.

[2] T. Cole, Thermoelectric energy conversion with solid electrolytes, Science 221 (1983) 915-920.

[3] J.M. Merrill, C. Mayberry, Experimental investigation of multi-AMTEC cell ground demonstration converter systems based on PX-3 and PX-5 series AMTEC cells, AIP Conf. Proc. 458 (1999) 1369-1377.

[4] N. Weber, A thermoelectric device based on beta-alumina solid electrolyte, Energy Convers. 14 (1974) 1-8.

[5] R.M. Williams, M.E. Loveland, B. Jeffries-Nakamura, M.L. Underwood, C. P. Bankston, H. Leduc, J.T. Kummer, Kinetics and transport at AMTEC electrodes: I. The interfacial impedance model, J. Electrochem. Soc. 137 (1990) 1709-1716.

[6] R.M. Williams, B. Jeffries-Nakamura, M.L. Underwood, C.P. Bankston, J. T. Kummer, Kinetics and transport at AMTEC electrodes: II. Temperature dependence of the interfacial impedance of $\mathrm{Na}(\mathrm{g}) /$ Porous Mo/Na-beta' alumina, J. Electrochem. Soc. 137 (1990) 1716-1723.

[7] M.T. Dunham, B.D. Iverson, High-efficiency thermodynamic power cycles for concentrated solar power systems, Renew. Sustain. Energy Rev. 30 (2014) $758-770$.

[8] A. Gunawan, A. Limia, J.M. Ha, P.A. Kottke, S.W. Lee, A.G. Fedorov, S.K. Yee, Techno-economic analysis of dual-stage sodium thermal electrochemical converter (Na-TEC) power block for distributed CSP, Proc. ASME Int. Conf. Energy Sustain. Paper No: ES2018-7505, V001T11A008; https://doi.org/10.1115/ES2018-7505.

[9] A. Limia, J.M. Ha, P. Kottke, A. Gunawan, A.G. Fedorov, S.W. Lee, S.K. Yee, A twostage sodium thermal electrochemical converter (Na-TEC), J. Power Sources 371 (2017) 217-224.

[10] J.M. Tournier, M.S. El-Genk, Performance analysis of Pluto/Express, multitube AMTEC cells, Energy Convers. Manag. 40 (1999) 139-173.

[11] S.P.S. Badwal, S.S. Giddey, C. Munnings, A.I. Bhatt, A. F Hollenkamp, Emerging electrochemical energy conversion and storage technologies, Front. Chem. 2 (2014) 79.

[12] H. Nakata, T. Nagata, K. Tsuchida, A. Kato, Ceramic electrodes for an alkali metal thermo-electric converter (AMTEC), J. Appl. Electrochem. 23 (1993) 1251-1258.

[13] M.A.K. Lodhi, A. Daloglu, Effect of geometrical variations on AMTEC cell heat losses, J. Power Sources 91 (2000) 99-106.

[14] M.A.K. Lodhi, P. Vijayaraghavan, A. Daloglu, An overview of advanced space/ terrestrial power generation device: AMTEC, J. Power Sources 103 (2001) 25-33.
[15] M.A.K. Lodhi, S.C. Soon, M. Mohibullah, Temperature-dependent grain growth model for AMTEC electrodes, J. Power Sources 135 (2004) 304-310.

[16] M.A.K. Lodhi, P. Vijayaraghavan, A. Daloglu, Time-dependent BASE performance and power degradation in AMTEC, J. Power Sources 93 (2001) 41-49.

[17] M. El Genk, J.M. Tournier, Recent advances in vapor-anode, multi-tube, alkali metal thermal-to-electric conversion cells for space power, Proc. Fifth Eur. Space Power Conf. 416 (1998) 257.

[18] W. Peng, X. Zhang, Z. Ye, J. Chen, Optimum operation states and parametric selection criteria of an updated alkali metal thermal electric converter, Energy Convers. Manag. 168 (2018) 230-236.

[19] S.Y. Wu, Y.C. Zhang, H. Yang, L. Xiao, Performance evaluation and parametric analysis of AMTEC/TEG hybrid system, Energy Convers. Manag. 154 (2017) $118-126$.

[20] W. Peng, W. Li, Z. Ye, G. Su, J. Chen, Parametric design strategies of an updated alkali metal thermoelectric converter-thermoelectric generator system operating at optimum states, Energy Convers. Manag. 182 (2019) 53-59.

[21] W. Peng, J. Gonzalez-Ayala, J. Guo, J. Chen, A.C. Hernández, An alkali metal thermoelectric converter hybridized with a Brayton heat engine: parametric design strategies and energetic optimization, J. Clean. Prod. 120953 (2020).

[22] S. Balagopal, A.V. Joshi, S. John, Multi-stage sodium heat engine for electricity and heat production, U.S. Patent Appl. No. 15/273,367.

[23] A. Limia, P. Kottke, A.G. Fedorov, S.K. Yee, Thermal modeling and efficiency of a two-stage sodium heat engine, Appl. Therm. Eng. 145 (2018) 603-609.

[24] B.J. De Cisneros, A.C. Hernández, Collective working regimes for coupled heat engines, Phys. Rev. Lett. 98 (2007) 130602.

[25] B.J. De Cisneros, A.C. Hernández, Coupled heat devices in linear irreversible thermodynamics, Phys. Rev. E 77 (2008), 041127.

[26] S.Y. Wu, L. Xiao, Y. Cao, Y.R. Li, A parabolic dish/AMTEC solar thermal power system and its performance evaluation, Appl. Energy 87 (2010) 452-462.

[27] J.M. Tournier, M.S. El-Genk, M. Schuller, P. Hausgen, An analytical model for liquid-anode and vapor-anode AMTEC converters, AIP Conf. Proc. 387 (1997) $1543-1552$.

[28] K. Deb, Multi-objective Optimization Using Evolutionary Algorithms, vol. 16, John Wiley \& Sons, 2001, 978-0-471-87339-6.

[29] C.A. Spence, M. Schuller, T.R. Lalk, Development, evaluation, and design applications of an AMTEC converter model, AIP Conf. Proc. 654 (2003) 685-700.

[30] J.M. Tournier, M.S. El-Genk, An electric model of a vapour anode, multitube alkali-metal thermal-to-electric converter, J. Appl. Electrochem. 29 (1999) 1263-1275. 